\title{
Alongwind Static Equivalent Wind Loads and Responses of Tall Buildings. Part I: Unfavorable Distributions of Static Equivalent Wind Loads
}

\author{
Yin Zhou*, Ming Gu, Haifan Xiang \\ State Key Lab. for Disaster Reduction in Civ. Eng., Dept. of Bridge Eng., Tongji University, Shanghai 200092, P.R. China
}

\begin{abstract}
Although on the same theoretical basis, the current standards of major countries have set out two evidently different distributions of along-wind static equivalent wind loads, one is the same as the mean wind force and the other is the same as the first mode shape, on tall buildings. In this paper, the fluctuating static equivalent wind load is evaluated as mean, background and resonant components, and the unfavorable distribution of each component is separately addressed. Meanwhile, the wind loads by the above two code methods are derived. The effects of the wind loads by the two code methods on tall buildings are then examined to identify whether or not they are equivalent with regard to the actual wind nduced responses. The results show that the wind loads by the two code methods can only ensure an equivalent first mode displacement response, and they may lead to some considerably unfavorable load effects, for example, the base shear force estimation. At last, a numerical example demonstrating the main results is given.
\end{abstract}

Keywords: Static equivalent wind load; "Gust loading factor" method; Tall building

\section{Introduction}

The along-wind responses of tall buildings under gusty wind are well known due to the primarily work of Dr. Davenport and other researchers in the early of 1960s; however, two voids associated with procedures to aid in the design still can be found in the literature to date: the first is on the distributions of the static equivalent wind loads; the second is on the effects of the mode shapes. Considerations of these two problems are separately the subjects of the present paper (part I) and a companion paper (part II).

The "gust loading factor" method ${ }^{[1]}$, originally introduced by Dr. Davenport (1967) as a classic method for computing the wind induced dynamic responses in along-wind, has been developed and applied in almost all the current standards of the major countries. The "gust loading factor" is defined as the ratio between the peak fluctuating displacement response and the mean displacement response. Because the mean wind force and the mean responses are generally appreciated by the engineers, the wind induced peak dynamic displacement response can easily be obtained when the gust loading factor is available. Besides the displacement response, the engineers also care for the internal forces, stresses and strains, etc.. It is more convenient to provide the static equivalent wind load for the engineering use.

However, the same attention to the "gust loading factor" has not been paid to the study of the static equivalent wind load to date. According to the "gust loading factor" method, the static equivalent wind load is equal to the mean wind force multiplied by the "gust loading

then the distribution of the equivalent wind load with the building height obeys $2 \alpha$ exponential law ( $\alpha$ is the exponent of the mean wind velocity profile), just as the mean wind force does. Among the current standards of the major countries and associations, the

\footnotetext{
*Corresponding author
} 
American Code ANSI/ASCE-93 ${ }^{[4]}$, Canadian Code NBC- $1995^{[5]}$ and the RLB-AIJ1993 ${ }^{[3]}$, etc., all provide this kind of $2 \alpha$ exponent wind load (hereafter referred to as GLF method). However, it has generally been recognized that the distribution of the resonant static equivalent wind load with the building height should obey $\beta$ exponential law ( $\beta$ is the exponent of the first mode shape; provided that the distribution of the mass along the building height is uniform). In general, $2 \alpha$ varies in the range of $0.2 \sim 0.7$, while $\beta$ is around 1 for typical tall buildings. So, the wind load by the GLF method is evidently different from the $\beta$ exponent resonant equivalent wind load. It is quite doubtful that the $2 \alpha$ exponent wind load by the GLF method can ensure the same effects as the actual values. On the other hand, the static equivalent wind load provided by the Chinese Loading Code, GBJ9-87 ${ }^{[6]}$, is equal to the inertial wind force producing the same first mode displacement response as the actual value, and obeys $\beta$ exponential law (hereafter referred to as GBJ code method). The Australian Code, AS1170.2-89 ${ }^{[7]}$, however, does not provide the static equivalent wind load and only gives a gust base moment effect factor. As far as the static equivalent wind load on the tall buildings is concerned, it seems that the misleading conception still exists.

In this paper, the fluctuating static equivalent wind load is evaluated as mean, background and resonant components according to the actual response characteristics of the tall buildings to gusty wind. The unfavorable distributions of these three components are separately set out. Meanwhile, the wind loads by the two code methods (GLF method and GBJ code method) are provided. Because of the evident difference of the wind loads distributions, the load effects of the wind loads by the two code methods are then examined to identify whether or not they are equivalent with regard to the actual wind induced responses of tall buildings. At last, a numerical example demonstrating the main results is given.

\section{Assumptions}

(i) The quasi-steady and strip theory are effective for the along-wind problem. The wind pressures in upwind and leeward are assumed to be fully correlated. ${ }^{[10]}$ The building is linear-elasticly responded to the gusty wind.

(ii) Uniform rectangular building, the width and the force coefficient are both constants along the height of the building

$$
B(z)=B, C_{d}(z)=C_{d} \text {. }
$$

(iii) The mass per unit height is constant in the vertical direction

$$
m(z)=m_{0} \text {. }
$$

(iv) Negligible resonant responses in modes higher than the first; The fundamental mode shape can be described by

$$
\varphi_{1}(z)=c(z / H)^{\beta}
$$

where $c, \beta$ are both constants.

(v) Mean wind velocity can be described by

$$
\bar{U}(z)=\bar{U}_{H}(z / H)^{\alpha}
$$

in which, $\bar{U}_{H}$ is the mean wind velocity evaluated at the top of the building, $\mathrm{H}$.

(vi) The spectrum describing the fluctuating wind is Davenport type spectrum; The RMS fluctuating wind velocity, $\sigma_{u}$, is constant with the height.

(vii) Negligible aerodynamic damping or other aero-elastic effects.

(viii) The coherence of the fluctuating wind pressure is slightly different from that in [1] as

$$
\begin{aligned}
& R_{x}\left(x_{1}, x_{2}, f\right)=\exp \left(-c\left|x_{1}-x_{2}\right|\right) ; R_{z}\left(z_{1}, z_{2}, f\right)=\exp \left(-c\left|z_{1}-z_{2}\right|\right) \\
& c=8 f h / \bar{U}(h), h=0.6 H
\end{aligned}
$$


in which, $\mathrm{h}$ is the reference height. ${ }^{[12]}$ Correspondingly, the joint acceptance functions in horizontal direction and vertical direction are separately defined as

$$
\begin{aligned}
& \left|J_{H}(f)\right|^{2}=\frac{1}{B^{2}} \int_{0}^{B} \int_{0}^{B} R_{x}\left(x_{1}, x_{2}\right) d x_{1} d x_{2} \\
& \left|J_{Z}(\alpha, \beta, f)\right|^{2}=\frac{(1+\alpha+\beta)^{2}}{H^{2}} \int_{0}^{H} \int_{0}^{H}\left(z_{1} / H\right)^{\alpha+\beta}\left(z_{2} / H\right)^{\alpha+\beta} R_{Z}\left(z_{1}, z_{2}\right) d z_{1} d z_{2} .
\end{aligned}
$$

(ix) The influence function of the building under externally applied or static equivalent loads is assumed to be in the form

$$
i(z)=i_{c}(z / H)^{\beta_{0}}
$$

where $i_{c}, \beta_{0}$ are both constants. The influence function is applicable to arbitrary load effects for instance, the internal forces, stress, deflection, and so on. $\beta_{0}$ is the exponent of influence function, and is usually different for different responses. For the base shear force and base moment responses, the coefficients are $i_{c}=1, \beta_{0}=0$ and $i_{c}=H, \beta_{0}=1$, respectively.

\section{Formulae of actual static equivalent wind loads and load effects}

In this section, the static equivalent wind load is separately evaluated as mean, background and resonant components. The latter two components are assumed to be un-correlated and are combined using a "root sum of squares" rule. ${ }^{[9]}$

\subsection{Mean wind force}

The mean wind force can be easily derived

$$
\bar{P}(z)=\frac{1}{2} \rho \bar{U}_{H}^{2} C_{d} B(z / H)^{2 \alpha} .
$$

Its effect on the structure is

$$
\begin{aligned}
\bar{r} & =\int_{0}^{H} \bar{P}(z) i(z) d z \\
& =1 / 2 \rho \bar{U}_{H}^{2} C_{d} B i_{c} \frac{H}{1+\beta_{0}+2 \alpha}
\end{aligned} .
$$

\subsection{Background component and its load effects}

The background responses at frequencies below the natural frequency are essentially quasi-static and the structure's response is not affected by its dynamic behavior. The background responses can be derived as ${ }^{[9]}$

$$
\begin{aligned}
& p(x, z)=\rho \bar{U}(z) u(x, z) C_{d} \\
& \hat{r}_{B}=g_{B}\left(\int_{0}^{\infty} \int_{0}^{H} \int_{0}^{H} \int_{0}^{B} \int_{0}^{B} \overline{p\left(x_{1}, z_{1}\right) p\left(x_{2}, z_{2}\right) i}\left(z_{1}\right) i\left(z_{2}\right) d x_{1} d x_{2} d z_{1} d z_{2} d f\right)^{1 / 2} \\
& \quad=\frac{g_{B} \rho \bar{U}_{H} C_{d} B H i_{c}}{\left(1+\alpha+\beta_{0}\right)}\left(\int_{0}^{\infty} S_{u}(f)\left|J_{H}(f)\right|^{2}\left|J_{Z}\left(\alpha, \beta_{0}, f\right)\right|^{2} d f\right)^{1 / 2}
\end{aligned}
$$

where $p(x, z)$ is the fluctuating wind pressure on the surface of the building at the point $(\mathrm{x}, \mathrm{z})$; the contribution of the second order term of the fluctuating wind velocity has been neglected.

The determination of the static equivalent wind load associated with the background response is somewhat difficult because of the correlation of the applied wind pressure. The "load response correlation" method, which was originally introduced by Kasperski et al. ${ }^{[8]}$, may be one of the effective approaches. If the correlation coefficient between the fluctuating 
wind load at height $\mathrm{z}$ and the load effect at height $z^{\prime}$ can be denoted by $Q(z)$, then the peak background load distribution can be given by

$$
\begin{aligned}
\hat{P}_{B}(z) & =g_{B} Q(z) \sigma_{p}(z) \\
Q(z) & =\frac{\int_{0}^{\infty} \int_{0}^{H} \int_{0}^{B} \int_{0}^{B} \overline{p\left(z, x_{1}\right) p\left(z_{1}, x_{2}\right)} i\left(z_{1}\right) d x_{1} d x_{2} d z_{1} d f}{\left(\int_{0}^{\infty} \int_{0}^{H} \int_{0}^{H} \int_{0}^{B} \int_{0}^{B} \overline{p\left(z_{1}, x_{1}\right) p\left(z_{2}, x_{2}\right) i}\left(z_{1}\right) i\left(z_{2}\right) d x_{1} d x_{2} d z_{1} d z_{2} d f\right)^{1 / 2} \cdot \sigma_{p}(z)}, \\
\sigma_{p}(z) & =\left(\int_{0}^{\infty} \int_{0}^{B} \int_{0}^{B}\left(\rho \bar{U}(z) C_{d}\right)^{2} S_{u}(f) R_{x}\left(x_{1}, x_{2}\right) d x_{1} d x_{2} d f\right)^{1 / 2} \\
& =\rho \bar{U}_{H} C_{d} B\left(\int_{0}^{\infty} S_{u}(f)\left|J_{H}(f)\right|^{2} d f\right)^{1 / 2}(z / H)^{\alpha}
\end{aligned}
$$

where $g_{B}$, the background peak factor, is assumed to be 3.7 in this paper; ${ }^{[7]} \sigma_{p}(z)$ is the RMS fluctuating externally applied wind force evaluated at height $\mathrm{z}$.

It can be seen from Eqs.13 15 that the distribution of the background equivalent wind load is independent of the dynamic behavior, but is dependent on the load effect concerned.

\subsection{Resonant component and its load effects}

Using methods of random vibration theory and spectral analysis, and white-noise excitation assumption, the first mode peak resonant displacement response can be computed from

$$
\hat{D}_{R 1}(z)=\frac{g_{R} \rho \bar{U}_{H} C_{d} B(2 \beta+1)}{m_{0}\left(2 \pi f_{1}\right)^{2}(1+\alpha+\beta)}\left|J_{H}\left(f_{1}\right) \| J_{Z}\left(\alpha, \beta, f_{1}\right)\right| \sqrt{\frac{\pi f_{1}}{4 \zeta_{1}} S_{u}\left(f_{1}\right)}\left(\frac{z}{H}\right)^{\beta} .
$$

The resonant equivalent fluctuating wind load can be represented by the inertial force

$$
\begin{aligned}
\hat{P}_{R 1}(z) & =m(z)\left(2 \pi f_{1}\right)^{2} \hat{D}_{R 1}(z) \\
& =\frac{g_{R} \rho \bar{U}_{H} C_{d} B(2 \beta+1)}{(1+\alpha+\beta)}\left|J_{H}\left(f_{1}\right) \| J_{Z}\left(\alpha, \beta, f_{1}\right)\right| \sqrt{\frac{\pi f_{1}}{4 \zeta_{1}} S_{u}\left(f_{1}\right)\left(\frac{z}{H}\right)^{\beta},} \\
g_{R}= & \sqrt{2 \ln \left(f_{1} T\right)}+\frac{0.577}{\sqrt{2 \ln \left(f_{1} T\right)}},
\end{aligned}
$$

in which, $f_{1}$ and $\zeta_{1}$ are the natural frequency and the critical damping ratio of the first mode, respectively. $\mathrm{T}$ is the observation time, and is set to be 600 seconds in this paper. It can be seen that the distribution of the resonant equivalent wind load obeys $\beta$ exponential law.

The load effect of the resonant equivalent wind load component is

$$
\begin{aligned}
\hat{r}_{R 1} & =\int_{0}^{H} \hat{P}_{R 1}(z) i(z) d z \\
& =\frac{g_{R} \rho \bar{U}_{H} C_{d} B H(2 \beta+1) i_{c}}{\left(1+\beta+\beta_{0}\right)(1+\alpha+\beta)}\left|J_{H}\left(f_{1}\right) \| J_{Z}\left(\alpha, \beta, f_{1}\right)\right| \sqrt{\frac{\pi f_{1}}{4 \zeta_{1}} S_{u}\left(f_{1}\right)} .
\end{aligned}
$$

\subsection{Resultant equivalent wind load and effects}

The mean wind force and the resonant equivalent wind load obey $2 \alpha$ and $\beta$ exponential law, respectively, and they are both independent of the response. Nevertheless, the distribution of the background equivalent wind load is not independent of the type of the response. It is suggested that the resultant peak load effects rather than the equivalent wind loads be combined in the following way

$$
\hat{r}(z)=\bar{r}(z)+\sqrt{\left(\hat{r}_{B}(z)\right)^{2}+\left(\hat{r}_{R 1}(z)\right)^{2}} .
$$




\section{Equivalent wind loads and effects based on the "gust loading factor" method}

As mentioned earlier, the equivalent wind load (or its components) by the GLF method is equal to the mean wind force multiplied by the gust factor (or its components). Based on this judgment, the equivalent wind load components by the GLF method are deduced in this section.

\subsection{Background component and its load effects}

The first mode mean displacement response, which is useful for the derivation of the formulae in this section, can be easily obtained

$$
\bar{D}_{1}(z)=\frac{2 \beta+1}{2 \alpha+\beta+1} \cdot \frac{\rho \bar{U}_{H}^{2} B C_{d}}{2 m_{0}\left(2 \pi f_{1}\right)^{2}}\left(\frac{z}{H}\right)^{\beta} .
$$

The first mode background displacement response can be computed from

$$
\hat{D}_{B 1}(z)=\frac{g_{B} \rho \bar{U}_{H} C_{d} B(2 \beta+1)}{m_{0}\left(2 \pi f_{1}\right)^{2}(1+\alpha+\beta)}\left(\int_{0}^{\infty} S_{u}(f)\left|J_{H}(f)\right|^{2}\left|J_{Z}(\alpha, \beta, f)\right|^{2} d f\right)^{1 / 2}\left(\frac{z}{H}\right)^{\beta} .
$$

The background equivalent wind load is equal to the mean wind force multiplied by the background gust factor, that is

$$
\begin{aligned}
\hat{P}_{B-2 \alpha}(z) & =G_{B} \bar{P}(z) \\
= & \frac{\hat{D}_{B 1}(z)}{\bar{D}_{1}(z)} \bar{P}(z) \\
= & \frac{g_{B} \rho \bar{U}_{H} C_{d} B(2 \alpha+\beta+1)}{(1+\alpha+\beta)}\left(\int_{0}^{\infty} S_{u}(f)\left|J_{H}(f)\right|^{2}\left|J_{Z}(\alpha, \beta, f)\right|^{2} d f\right)^{1 / 2}\left(\frac{z}{H}\right)^{2 \alpha}
\end{aligned}
$$

The arbitrary peak background response can be derived as

$$
\begin{aligned}
\hat{r}_{B-2 \alpha} & =\int_{0}^{H} \hat{P}_{B-2 \alpha}(z) i(z) d z \\
& =\frac{g_{B} \rho \bar{U}_{H} C_{d} B H(2 \alpha+\beta+1) i_{c}}{\left(1+2 \alpha+\beta_{0}\right)(1+\alpha+\beta)}\left(\int_{0}^{\infty} S_{u}(f)\left|J_{H}(f)\right|^{2}\left|J_{Z}(\alpha, \beta, f)\right|^{2} d f\right)^{1 / 2} .
\end{aligned}
$$

\subsection{Resonant component and its load effects}

Similarly, with the first mode resonant displacement response (Eq.16), the resonant equivalent wind load can be obtained

$$
\begin{aligned}
& \hat{P}_{R-2 \alpha}(z)=G_{R} \bar{P}(z) \\
& =\frac{\hat{D}_{R 1}(z)}{\bar{D}(z)} \bar{P}(z) \\
& =g_{R} \rho \bar{U}_{H} C_{d} B \frac{(1+\beta+2 \alpha)}{(1+\alpha+\beta)}\left|J_{H}\left(f_{1}\right)\right|\left|J_{Z}\left(\alpha, \beta, f_{1}\right)\right| \sqrt{\frac{\pi f_{1}}{4 \zeta_{1}} S_{u}\left(f_{1}\right)}\left(\frac{z}{H}\right)^{2 \alpha}
\end{aligned}
$$

The arbitrary resonant response can be computed from

$$
\begin{aligned}
\hat{r}_{R-2 \alpha} & =\int_{0}^{H} \hat{P}_{R-2 \alpha}(z) i(z) d z \\
& =\frac{g_{R} \rho \bar{U}_{H} C_{d} B H(1+2 \alpha+\beta) i_{c}}{\left(1+2 \alpha+\beta_{0}\right)(1+\alpha+\beta)}\left|J_{H}\left(f_{1}\right)\right|\left|J_{Z}\left(\alpha, \beta, f_{1}\right)\right| \sqrt{\frac{\pi f_{1}}{4 \zeta_{1}} S_{u}\left(f_{1}\right)} .
\end{aligned}
$$

\section{Equivalent wind loads and effects by the GBJ code method}


The dynamic equivalent wind load (or its components) by the GBJ code method is equal to the inertial wind force producing the same first mode displacement response as the actual value. It means that the resonant equivalent wind load and effects by the GBJ code method are equal to the actual ones or Eqs.17 and 19. But the background component is different, which is derived in this section.

With the first mode background displacement response (Eq.22), the background equivalent wind load by the GBJ code method can be easily obtained

$$
\begin{aligned}
\hat{P}_{B-\beta}(z) & =m_{0}\left(2 \pi f_{1}\right)^{2} \hat{D}_{B 1}(z) \\
& =\frac{g_{B} \rho \bar{U}_{H} C_{d} B(2 \beta+1)}{(1+\alpha+\beta)}\left(\int_{0}^{\infty} S_{u}(f)\left|J_{H}(f)\right|^{2}\left|J_{Z}(\alpha, \beta, f)\right|^{2} d f\right)^{1 / 2}\left(\frac{z}{H}\right)^{\beta} .
\end{aligned}
$$

The arbitrary background response can then be computed from

$$
\begin{aligned}
\hat{r}_{B-\beta} & =\int_{0}^{H} \hat{P}_{B-\beta}(z) i(z) d z \\
& =\frac{(2 \beta+1) g_{B} \rho \bar{U}_{H} C_{d} B H i_{c}}{\left(1+\beta+\beta_{0}\right)(1+\alpha+\beta)}\left(\int_{0}^{\infty} S_{u}(f)\left|J_{H}(f)\right|^{2}\left|J_{Z}(\alpha, \beta, f)\right|^{2} d f\right)^{1 / 2} .
\end{aligned}
$$

\section{Deviation factors of the load effects and parametric analysis}

Except for the mean wind load, the distributions of the background and resonant equivalent wind load components by the two code methods are different from those of the actual ones as indicated in the above sections. It is necessary to study the effects of the loads on the responses to identify whether or not they are equivalent with regard to the actual wind induced responses. In this section, the effects of background and resonant wind load components are separately examined.

\subsection{Background response deviation factors}

The background response deviation factor, $C_{B}$, are defined as the ratio of the load effects of the background equivalent wind loads by the two code methods to the actual background response, and they can be deduced using Eqs.12, 24 and 28 as

$$
\begin{aligned}
C_{B-2 \alpha}= & \hat{r}_{B-2 \alpha} / \hat{r}_{B} \\
= & \frac{(1+2 \alpha+\beta)\left(1+\alpha+\beta_{0}\right)\left(\int_{0}^{\infty} S_{u}(f)\left|J_{H}(f)\right|^{2}\left|J_{Z}(\alpha, \beta, f)\right|^{2} d f\right)^{1 / 2}}{\left(1+2 \alpha+\beta_{0}\right)(1+\alpha+\beta)\left(\int_{0}^{\infty} S_{u}(f)\left|J_{H}(f)\right|^{2}\left|J_{Z}(\alpha, \beta, f)\right|^{2} d f\right)^{1 / 2}} . \\
C_{B-\beta}= & \hat{r}_{B-\beta} / \hat{r}_{B} \\
= & \frac{(1+2 \beta)\left(1+\alpha+\beta_{0}\right)\left(\int_{0}^{\infty} S_{u}(f)\left|J_{H}(f)\right|^{2}\left|J_{Z}(\alpha, \beta, f)\right|^{2} d f\right)^{1 / 2}}{\left(1+\beta+\beta_{0}\right)(1+\alpha+\beta)\left(\int_{0}^{\infty} S_{u}(f)\left|J_{H}(f)\right|^{2}\left|J_{Z}\left(\alpha, \beta_{0}, f\right)\right|^{2} d f\right)^{1 / 2}} .
\end{aligned}
$$

For the first mode displacement response the exponent of the influence function $\beta_{0}=\beta$ (refer to Eqs.16, 21 and 22), the background factor $C_{B-2 \alpha}=C_{B-\beta}=1$ regardless of the values of other parameters. It means that the first mode background displacement responses by both the GLF method and the GBJ code method are equivalent to the actual value.

The sensitivity of background deviation factor of the GLF method, $C_{B-2 \alpha}$, to its parameters is illustrated in Figure 1. 


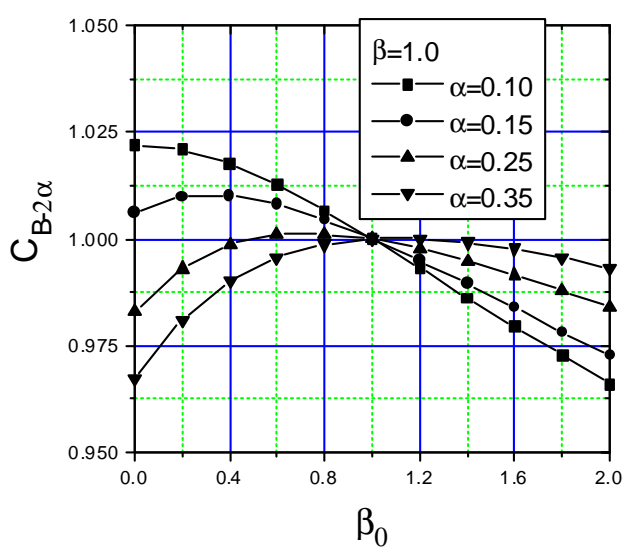

(a)

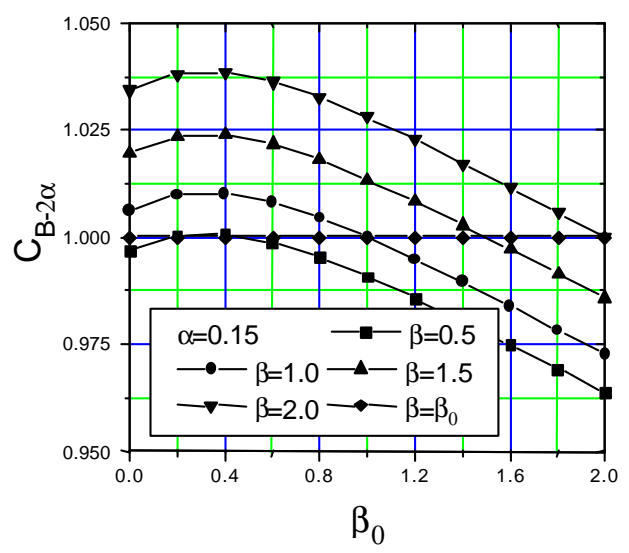

(b)

Figure 1. Background response deviation factor of GLF method

It can be seen from Fig. 1 that the background deviation factor of the GLF method is sensitive to the parameter $\beta_{0}$ (i.e. the type of the response). In Fig.1 (a), $\beta=1$, which is an assumption generally adopted in most codes. The background factor is relatively disperse with regard to its parameters, but ranges within a rather small region of $0.96 \sim 1.03$. It seems that the $2 \alpha$ exponent background equivalent wind load by the GLF method is a fairly good approximation of the actual response dependent background wind loads. In Fig. 1 (b), $\alpha=0.15$, and $\beta$ varies. The background factor varies in a slightly wider region than that for $\beta=1$, but still within $5 \%$.

The sensitivity of background deviation factor of the GBJ code method, $C_{B-\beta}$, to its parameters is illustrated in Figure 2.

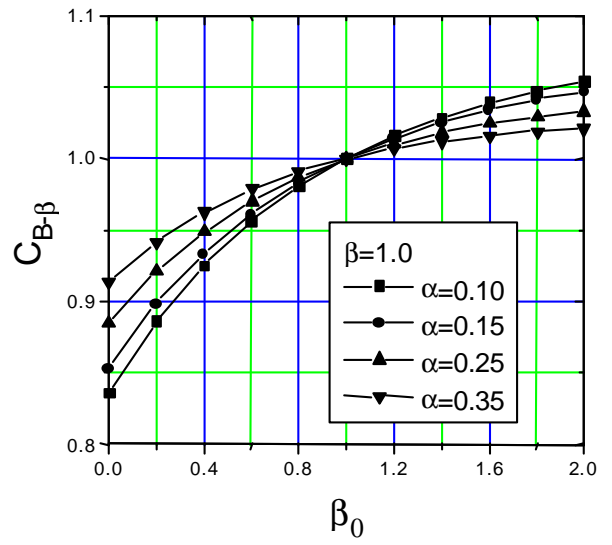

(a)

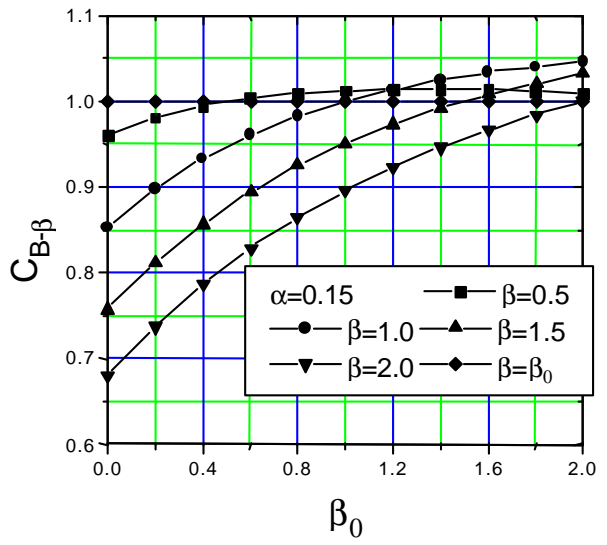

(b)

Figure 2. Background response deviation factor of GBJ code method

From Fig.2, it can be seen that the background deviation factor of the GBJ code method is also sensitive to parameter $\beta_{0}$. In Fig.2(a), $\beta=1$. The background factor ranges from 0.84 to 1.05 as $\alpha$ varies from 0.1 to 0.35 . In Fig.2(b), $\alpha=0.15$, and $\beta$ varies. The background factor varies in a wider region than that for $\beta=1$ of $0.67 \sim 1.05$ as $\beta$ from 0.5 to 2.0 . When $\alpha=0.15$, as far as the base shear force response is concerned, the background factor is 0.85 as $\beta=1$, and 
0.67 as $\beta=2.0$. It means that the equivalent wind load by the GBJ code method will lead to unsafe background responses. For the majority of structures, the background responses will be dominant ${ }^{[5]}$ the GBJ code method will correspondingly result in unsafe design.

It should be pointed out that, because the background factor is related not only to the parameters $\alpha, \beta, \beta_{0}$, but to the definitions of the wind spectrum and correlation of the wind pressure, when the latter definitions are different from those adopted in this paper, the factor will be somewhat different. Another should be pointed out is that, the integration region adopted in the computation of the response is $0 \sim \mathrm{H}$. For some kinds of responses, such as the shear forces in the middle stories, this would not certainly be an unfavorable region. ${ }^{[1]}$

\subsection{Resonant response deviation factor}

As mentioned earlier, the resonant equivalent wind load and effects by the GBJ code method are the same as the actual values. Only the resonant response deviation factor of the GLF method, $C_{R-2 \alpha}$, is addressed here. Using Eqs.19 and 26, the factor can be deduced as

$$
\begin{aligned}
C_{R-2 \alpha} & =\hat{r}_{R-2 \alpha} / \hat{r}_{R 1} \\
& =\frac{(1+2 \alpha+\beta)\left(1+\beta+\beta_{0}\right)}{\left(1+2 \alpha+\beta_{0}\right)(1+2 \beta)} .
\end{aligned}
$$

Like that for the background factor, the resonant factor $C_{R-2 \alpha}=1$ when $\beta_{0}=\beta$. That is to say, the resonant equivalent wind load given by the GLF method is also equivalent with regard to the first mode displacement response.

The sensitivity of the resonant factor to its parameters is illustrated in Figure 3.

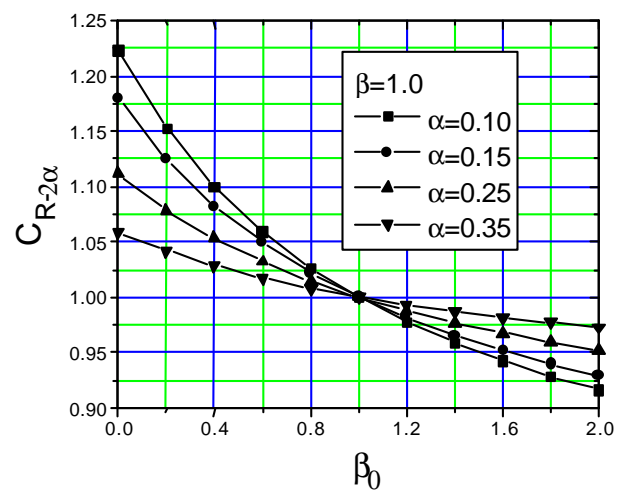

(a)

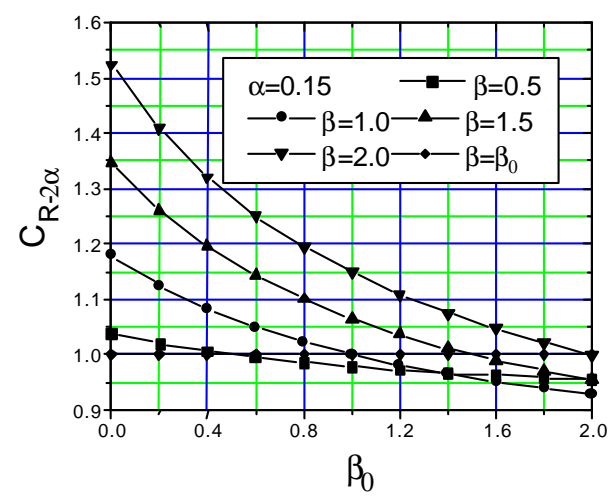

(b)

Figure 3. Resonant response deviation factor of GLF method

In Fig.3(a), $\beta=1$. The resonant factor of the GLF method is also sensitive to parameter $\beta_{0}$ but varies in a wider range than the corresponding background factor of 0.92 1.23. The resonant factor approaches gradually 1 as $\alpha$ increases, which implies a closer wind load to the actual resonant equivalent wind load. For the base shear force $\left(\beta_{0}=0\right)$, the factor is as high as 1.23 when $\alpha=0.10$, which can hardly be neglected. In Fig.3(b), $\alpha=0.15$ and $\beta$ varies. The resonant factor is more sensitive to $\beta$ than to $\alpha$, and varies in a wider range than that in (a) of $0.93 \sim 1.52$ as $\beta$ from 0.5 to 2.0. For the base shear force response, the factor is 1.18 when $\beta=1.0$, while it is 1.52 when $\beta=2.0$. The effect of nonlinear mode shape $(\beta \neq 1)$ will be examined in a the companion paper (part II). 


\section{Numerical example}

An assumed tall building, $\mathrm{H} * \mathrm{~B} * \mathrm{D}=200 * 50 * 40 \mathrm{~m}$ with 50 floors. The mass per unit height is $m_{0}=234275 \mathrm{~kg} / \mathrm{m}$. Its first mode frequency is $0.2 \mathrm{~Hz}$, the critical damping ratio is 0.01 . The reference wind velocity at $10 \mathrm{~m}$ height is $30 \mathrm{~m} / \mathrm{s}$, the exponent of the wind velocity profile is $\alpha=0.15$, the intensity of the turbulence wind evaluated at $10 \mathrm{~m}$ height is $\sigma_{u} / \bar{U}_{10}=0.2$.

In this section, the background equivalent wind loads for the base shear force, the base moment and the first mode displacement response are computed with the "L.R.C. Method", the background equivalent wind loads by the two code methods are als o computed. Meanwhile, mean wind force, resonant equivalent wind load of actual and by the GLF method are also provided. The distributions of the above loads and the distributions of the shear force, moment and displacement responses under the action of those loads are separately illustrated in Figure 4 and Figure 5.

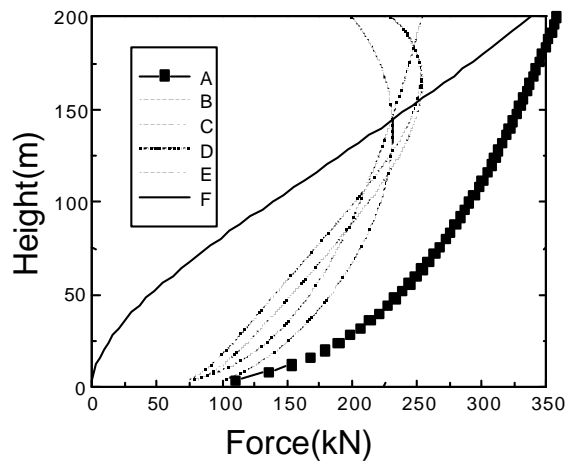

(a)

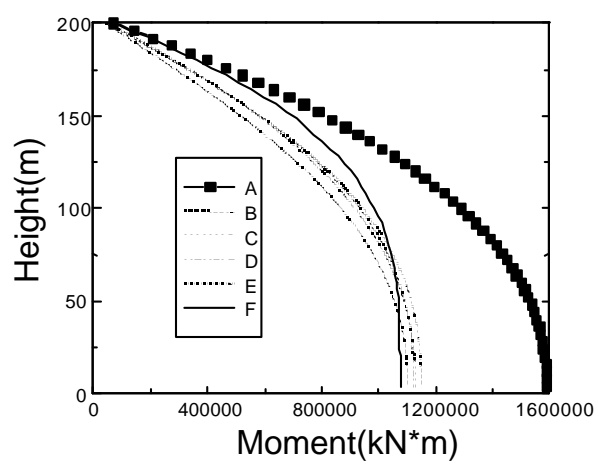

(c)

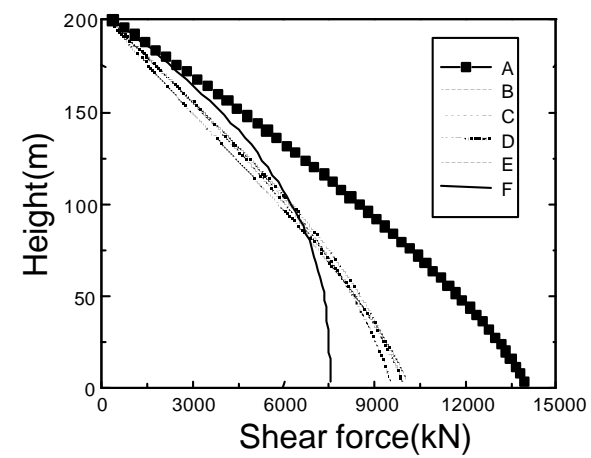

(b)

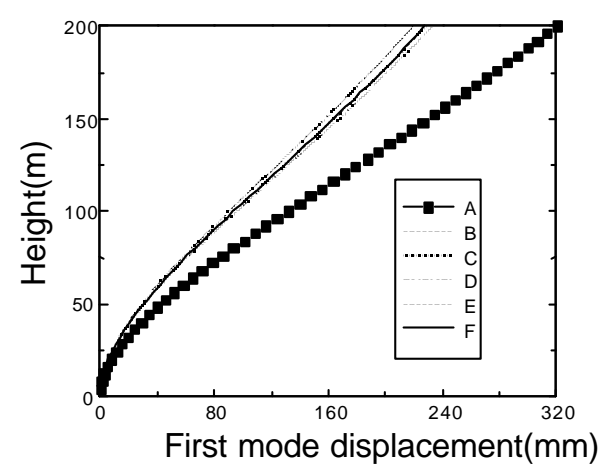

(d)

A: Mean wind force; B: Background equivalent wind load for the base shear force; C: Background equivalent wind load for the base moment; D: Background equivalent wind load for the first mode displacement response; E: Background equivalent wind load by the GLF method; F: Background equivalent wind load by the GBJ code method

Figure 4. Background equivalent wind loads and their shear force, moment, displacement responses 


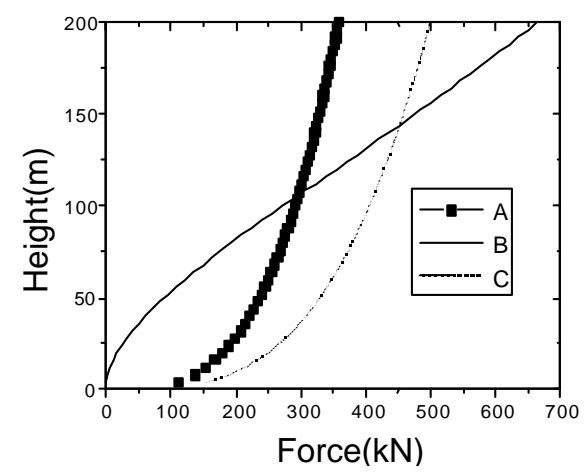

(a)

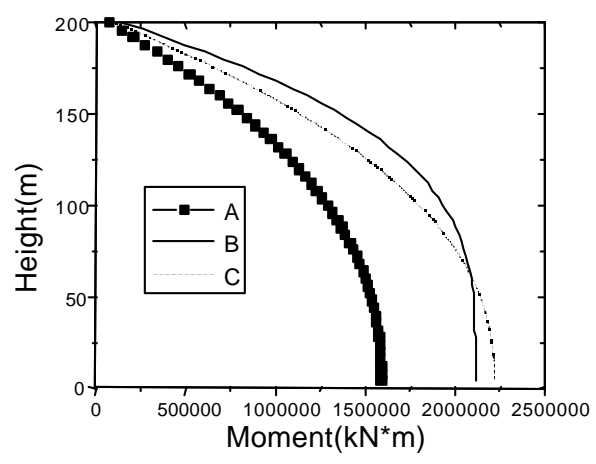

(c)

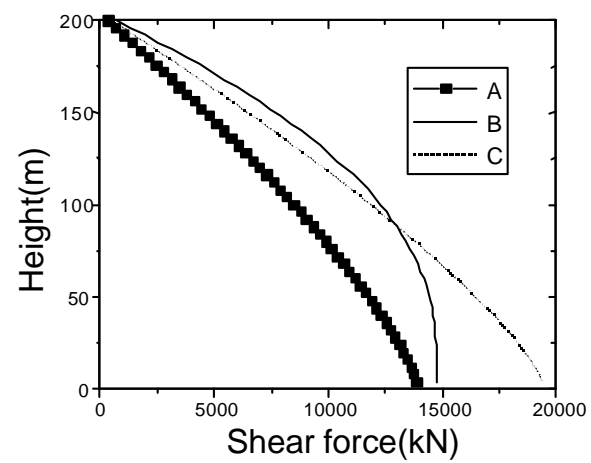

(b)

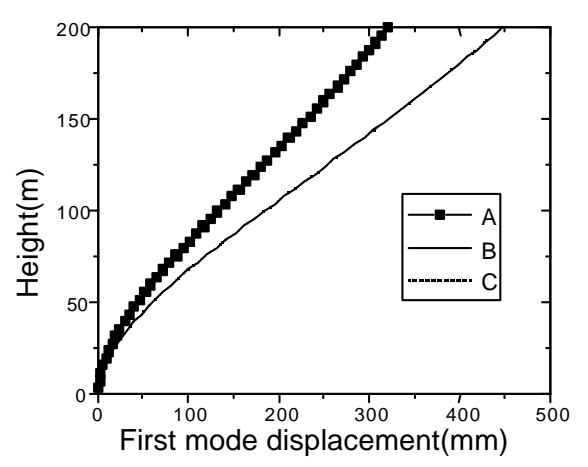

(d)

A: The mean wind force; B: The resonant equivalent wind load of actual value or by the GBJ code method; C: The resonant equivalent wind load by the GLF method;

\section{Figure 5. Resonant equivalent wind loads and their shear force, moment, and displacement responses}

Define the "gust effect factor" as the ratio between the resultant peak response of arbitrary type $\hat{r}$ (Eq.20) and the mean value $\bar{r}$ (Eq.10). Considering the factors for the base shear force, base moment, and the first mode displacement responses, the results for this numerical example with different methods are listed in Table 1.

Table 1. Gust effect factor

\begin{tabular}{cccc}
\hline & Base shear force & Base moment & First mode displacement \\
Method of this paper & 2.29 & 2.51 & 2.57 \\
GLF method & 2.57 & 2.57 & 2.57 \\
GBJ method & 2.19 & 2.49 & 2.57 \\
\hline
\end{tabular}

It can be concluded from this numerical example that:

(1) The distributions of the background equivalent wind loads for different responses computed by the L.R.C. Method are different from each other, and they are also different from those given by the two code methods. Meanwhile, the $2 \alpha$ exponent resonant equivalent wind load given by the GLF method is also evidently different from that given by the GBJ code method or the actual $\beta$ exponent resonant equivalent wind load.

(2) Different load effects have been obtained as expected resulting from the different distributions of equivalent wind loads. Although the first mode displacement response is 
identical between the three kinds of equivalent wind loads, the conclusion can not be extended to other load effects. For this numerical example, as far as the base shear force response is concerned, the background responses by GLF method and GBJ code method and the resonant response by GLF method are separately $98.2 \%, 74.8 \%$ and $131.4 \%$ of the actual values, the latter two can hardly be neglected even from the engineering viewpoint.

(3) The gusty effect or the gust effect factor is different for a given building depending on which response is concerned. However, the gust effect factor by the GLF method for any kind of response is the same, namely the "gust loading factor" or the first mode displacement gust effect factor, which will inevitably result in inconsistencies. For the base shear force response of this example, the gust effect factor by the GLF method is $12 \%$ more than the actual value.

(4) Although the background base shear force response by the GBJ code method is $25.2 \%$ lower than the actual value, the resultant response is only $4.4 \%$ less because for this numerical example the resonant response is dominant.

(5) The background responses under the action of the background equivalent wind loads computed by the L.R.C. method are truly most unfavorable, which confirms the effectiveness of the L.R.C. method. Furthermore, the adoption of the L.R.C. method in this paper can also ensure a consecutive transition of the formulae suitable for buildings from relative low or rigid to high and flexible.

\section{Conclusions}

In this paper, the fluctuating static equivalent wind load is separately evaluated as mean, background and resonant components according to the actual response characteristics of the tall buildings to gusty wind. The background equivalent wind load component is derived with the L.R.C. method, while the resonant component is represented by the inertial wind force. Meanwhile, these components are also provided with two code methods, namely the GLF method and the GBJ code method. Because of the evidently different distributions of the equivalent wind loads given by different methods, the effects of the equivalent wind loads on the responses are then examined to identify whether or not they are equivalent with regard to the actual wind induced responses of tall buildings. The results show that the wind loads by the two code methods can only ensure an equivalent first mode displacement response, while they may lead to some considerably unfavorable load effects, for example, the base shear force estimation.

Although the resonant equivalent wind load component is the same as the actual value, the background component by the GBJ code method is not identical to the actual value, which will lead to unsafe response estimation. For the majority of structures the background responses will be dominant. Because the GBJ code method may result in unsafe design, it is not suitable for code use.

Neither the background nor the resonant component by the GLF method is identical to the actual wind load. Because the deviation of the background responses is generally within 5\%, the background component by the GLF method is a fairly good approximation for the actual response dependent equivalent wind loads. However, the same conclusion can not be extended to the resonant component which may lead to some considerably unfavorable resonant response, for example, the base shear force estimation. When the building is high, flexible or with low damping, the resonant response will be dominant. The GLF method will then result in excessively conservative design and is not suitable for code use.

The distributions of the mean wind force and the resonant equivalent component obey respectively $2 \alpha$ and $\beta$ exponent law. The distribution of the background component is dependent on the load effect concerned, but it can be well approximated with the $2 \alpha$ exponent first mode displacement equivalent wind load. With these load components, the corresponding 
load effect components can easily be obtained and then be combined to the resultant effect using Eq.20 in this paper.

\section{Acknowledgment:}

The research work in this paper is jointly supported by the Chinese National Science Foundation and by the Chinese National Science Foundation for Outstanding Youth Scientists. This support is gratefully acknowledged. The work is also partially under a Joint Research Program with Fujita Corporation of Japan, the authors would like to express appreciation to the Corporation for the financial support, as well as to Dr. M. Nakayama of the Corporation for his kindly supply of some relevant materials.

\section{Appendix I. References:}

[1]. Davenport A.G., "Gust loading factors", Journal of the Structural Division, ASCE, Vol.93, No.ST3, June 1967, PP.11-34

[2]. Simiu E., Scanlan R.H., Wind Effects on Structures: An Introduction to Wind Engineering (2nd ed. ), John Wiley \& Sons, New York, 1986

[3]. Architectural Institute of Japan, Recommendations for loading on buildings, RLB-AIJ1993 ( in English version ), 1996

[4]. ASCE, Minimum Design Loading for Buildings and Other Structures, ANSI/ ASCE7-93, 1994

[5]. NRCC, User's Guide-NBC1995 Standard Commentaries, 1996

[6]. Chinese loading code GBJ9-87, 1990 (in Chinese)

[7]. Australian Standard, AS1170.2-89, SAA Loading Code, 1989

[8]. Kasperski M., Niemann H. J., "The L.R.C. method- a general method of estimating unfavorable wind load distributions for linear and non-linear structures", J. of Wind Engineering \& Industrial Aerodynamics, 41-44(1992)1753-1763

[9]. Davenport A.G., "How can we simplify and generalize wind loading?", J. of Wind Engineering \& Industrial Aerodynamics, 54/55(1995)657-669

[10].Kareem A., "Synthesis of fluctuating along wind loads on buildings", J. of Engrg. Mech., ASCE, Vol.112, 1986

[11].Holmes J.D., "Along-wind response of lattice towers- III. Equivalent load distribution", Engineering Structures, Vol. 18, No. 7, PP.489-484,1996

[12].Solari G., "Gust buffeting. I: Peak wind velocity and equivalent pressure", J. of Struct. Engrg., ASCE, Vol.109, No.2, 1993, PP.365 382

\section{Appendix II. Notation}

The following symbols are used in this paper:

$$
\begin{aligned}
& \hat{Y}(z), \bar{Y}(z)= \text { the peak and average displacement response } \\
& \bar{D}_{1}(z), \hat{D}_{B 1}(z), \hat{D}_{R 1}(z)=\begin{array}{l}
\text { the average, peak background and peak resonant first mode } \\
\text { displacement response }
\end{array} \\
& \hat{P}(z), \bar{P}(z)=\begin{array}{l}
\text { the peak and average wind force } \\
\text { the peak background equivalent wind load by the GLF method, }
\end{array} \\
& \hat{P}_{B-2 \alpha}(z), \hat{P}_{B-\beta}(z), \hat{P}_{B}(z)=\begin{array}{l}
\text { GBJ code method and of the actual value } \\
\hat{P}_{R-2 \alpha}(z), \hat{P}_{R}(z)=
\end{array} \quad \begin{array}{l}
\text { the peak resonant equivalent wind load by the GLF method and } \\
\text { of actual value }
\end{array}
\end{aligned}
$$




$$
\begin{aligned}
H, B, D & =\text { the height, width and the depth of the building } \\
C_{D}(z) & =\text { drag force coefficient } \\
m(z) & =\text { the mass per unit height } \\
\varphi_{1}(z) & =\text { the first mode shape } \\
c, \beta & =\text { constants, normalization factor and exponent of the first mode } \\
i(z) & =\text { shape } \\
i_{c}, \beta_{0} & =\text { confluence function } \\
\bar{U}(z) & =\text { average wind velocity } \\
\alpha & =\text { exponent of average wind velocity profile } \\
u(z), \sigma_{u}, S_{u}(f) & =\text { fluctuating wind velocity, its RMS value and spectrum } \\
p(z), \sigma_{p}(z) & =\text { fluctuating wind pressure and its RMS value } \\
R_{x}, R_{z} & =\text { coherence of the applied wind pressure } \\
J_{H}, J_{Z} & =\text { joint acceptance function in horizontal and vertic al direction } \\
\bar{r}, \hat{r}_{B}, \hat{r}_{R}, \hat{r}= & \text { mean, peak background, peak resonant and peak resultant } \\
G_{B}, G_{R}, G= & \text { background, resonant component of gust factor and the gust } \\
g_{B}, g_{R}= & \text { bactor } \\
Q(z)= & \text { load response correlation factor } \\
f_{1}, \zeta_{1} & =\text { frequency and critical damping ration in the first mode } \\
\mathrm{T} & =\text { observation time } \\
C_{B-2 \alpha}, C_{R-2 \alpha}= & \text { background and resonant response deviation factors of the GLF } \\
C_{B-\beta}= & \text { method }
\end{aligned}
$$

